\title{
Peruvian maca and possible impact on fertility
}

\begin{abstract}
Introduction: Maca (Lepidium meyenii) belongs to the Brassicaceae family and it grows in Peru's central Andes above 4000 and 4500meters of altitude from the sea level. The plant belongs to the brassica (mustard) family and the Lepidium genus. Its closest relatives are rapeseed, mustard, turnip, and cabbage. Maca has multiple clinical applications like proactive against cancer, libido maintenance, menopause, and fertility. There are several mechanisms the Maca could impact at fertility problems. The increase of Luteinizing hormone (LH), increase the semen quality and improve sexual development in male, and at female, increases the libido and improve menopause symptoms with a possible influence at germ cell quality.
\end{abstract}

Objectives: To describe the impact of consuming Maca involve an improve at fertility.

Methods: Literature search was performed in PubMed from January 2000 to May 2017 using the search terms child obesity and: infertility, Maca, ovulation, spermatogenesis.

Conclusion: Maca consumption can improve the quality of both germs cells enlightening the fertility. More clinical studies are need to know the doses and direct the effect of the microenvironment in germ cells having a positive impact on reproduction in both genders.

Keywords: maca, fertility, germ cells
Volume 6 Issue 5 - 2017

\author{
José Manuel Lozano Sánchez,' Zoraida \\ Axtle Serrano,' Julio Avilés Durán, ${ }^{2}$ Héctor \\ Salvador Godoy Morales, ${ }^{2}$ Paola Berenice \\ Merchand Álvarez, ${ }^{2}$ Ricardo Mera Mejía, ${ }^{2}$ \\ Luis Felipe Montaño Estrada, ${ }^{3}$ Erika Patricia \\ Rendón Huerta, ${ }^{3}$ \\ 'CSIHM Centro de Salud Integral del Hombre y la mujer, \\ Mexico \\ ${ }^{2}$ ART Reproduccion, México \\ ${ }^{3}$ Faculty of Medicine, Universidad Nacional Autónoma de \\ México, Mexico
}

Correspondence: Zoraida Axtle Serrano, Department of Nutrition and Metabolism, CSIHM Centro de Salud Integral del Hombre y la mujer, Tehuantepec 25 I cons 506, México City, México, Tel: (0II)5541652834; Email: draaxtle@csihm.com

Received: June 05, 2017| Published: June 12, 2017

\section{Introduction}

Maca (Lepidium meyenii) belongs to the Brassicaceae family and it grows in Peru's central Andes above 4000 and 4500 meters of altitude from the sea level. The plant belongs to the brassica (mustard) family and the Lepidium genus. Its closest relatives are rapeseed, mustard, turnip, and cabbage.

The underground part of the plant, the tuber, is the main product used for human consumption. There are several varieties, and its major phenotypes are yellow, red, and black and it seems that depends of the type of soil where it is cultivated. ${ }^{1}$ The biologic activity of the plant is in the hypocotyl which grows inside the land as a bulbous material. Its potential bioactive ingredients in maca include macadirine, macamides, macaene, glucosinolates, maca alkaloid and maca nutrients. ${ }^{2}$ Glucosinolates are the most important metabolites in maca because of their biological activity against cancer. Alkaloids have been studied as the main stimulators of ovarian follicles on female rats and enhancing spermatogenesis on males, increasing spermatozoids on seminiferous tubes and mitosis. ${ }^{3}$

Several articles describe its wide clinical use, like improving sexual function, treatment of neurological disorders such as anxiety, depression, and memory loss. The most studied effect is the enhancing of fertility and as an aphrodisiac. The focus of several studies is to improve the symptoms of menopause, reproduction, and hormone balance on female. In male, the improvement of sperm function, nevertheless the level of evidence is limited.

\section{Lepidium meyenii on spermatogenesis}

Black maca increases spermiation stages (VII-VIII) and germinal cells mitosis (IX-XI). Such as the daily sperm production and epididymal count of sperm without affecting testosterone, luteinizing hormone (LH) and follicle stimulating hormone (FSH) levels. Maca may also regulate apoptosis in these stages increasing the number of sperm released during spermiation. This may explain why epididymal sperm count is increased as early as three days after treatment with maca. ${ }^{4}$

The lack of testosterone response suggests that bioavailable testosterone or testosterone receptor binding might be augmented. Maca may also acts without participation of androgen mechanism. As can be seen in the seminal vesicle is not influenced by Maca, a target for androgen action. ${ }^{5}$

Studies suggest that maca regulates sperm count by maintaining the balance between oxidant and antioxidant status. ${ }^{6}$ The aphrodisiac activity has been related to its lipidic fraction, which contains mainly fatty acids and macamides. These are used to assess the quality of maca products. ${ }^{\text {? }}$

Probably the most relevant studies on the effects of maca on testicular gene expression for luteinizing hormone receptor, steroidogenic acute regulatory protein, and steroidogenic enzymes determines that maca increases testicular mRNA level for $3 \beta$-hydroxysteroid dehydrogenase $(3 \beta-H S D)$ an enzyme related to androgen production. Enhanced activity of $3 \beta-\mathrm{HSD}$ could increase androstenedione, a precursor of testosterone, increasing production of testosterone, this also may be related to changes in the activity of reactive oxygen species. ${ }^{3}$ A recent study ${ }^{8}$ where they feed maca extracts for long period times stated the same results of transient testosterone increase by Leydig cells, suggesting that maca may alleviate the decline in steroidogenic ability of Leydig cells with ageing. ${ }^{8}$ The ecotypes have different biological effects.

Reduction of prostate weight was significantly reduced by the administration of Red Maca whereas the Black and Yellow Maca 
did not present this effect. Suggesting that Red Maca prevented the increase in prostate size. They also showed an increase in daily sperm production after long and short term treatment (7 to 42days) with Black Maca. Yellow Maca increased epididymal sperm count. Black Maca increased daily sperm production and efficiency, suggesting that Black Maca may become a potential treatment for male infertility. ${ }^{9}$ Aqueos extract of black maca improved memory impairment by its antioxidant and acetyl cholinesterase inhibitory activities. The antioxidant capacity of maca has been demonstrated. Increased cell viability and decreased cell cytotoxicity in cells pretreated with the extract of maca leaves confirms it. ${ }^{10}$

\section{Lepidium meyenii on female fertility}

Maca promotes optimal functioning of the hypothalamus and the pituitary, thereby improving the functioning of all the endocrine glands. It has been recorded the balance effects on FSH, estradiol and progesterone. Also, restricting weight increase, lowering triglycerides in blood plasma and increasing phosphorus and calcium deposition in bone and muscle tissues.

The alkaloids present in Maca are non-steroid compounds, which are characterized by better tolerance by women who use it. It's well documented that maca stimulates or contributes to the regulatory mechanism for secretion of the quantity of estrogen above $30 \mathrm{pg} / \mathrm{ml}^{8}{ }^{8}$ reducing menopausal discomfort. Simultaneously had a distinctive tendency to lower FSH. Maca appears to have positive effect on depression with a sedative effect as well. This effect has been associated with lowering cortisol and ACTH levels. ${ }^{11}$

Administration of Maca has been reported to increase serum levels of FSH, estradiol, progesterone, and ACTH on perimenopausal women. Also, the symptoms on menopausal women seem to be improved. ${ }^{12}$ Other studies have demonstrated that Maca intake enhances LH serum levels during the LH surge but not the pulsate phase in female rats. This effect promotes ovulation trough pituitary function of the Hypophysis Pituitary Gonad axis. Supporting the traditional use of Maca to enhance fertility. ${ }^{13}$ There are several studies describing fertility improvement when Maca is administered by eliminating free radicals and generating an anti-oxidant function, mainly because of its metabolites the alkaloids and glycosylates. This and the enhancing of LH serum levels may be the mechanisms that improve fertility..$^{14,15}$

In each study that describes the effects on menopause symptoms, suggest that Maca is more effective than placebo. Most of them lack rigorous trial exams and are burdened with methodological flaws. As well, the clinical heterogeneity of some trials didn't allow a clear interpretation of the results as others provided insufficient data.

\section{Conclusion}

Peruvian Maca (Lepidium meyenii) have several possible uses, as we mention for improving sexual function, and fertility by impact of hormones levels specifically Luteinizing Hormone (LH) concentrations. With that the Maca consumption improves at female menopause, reproduction, and hormone balance. In male, the improvement of sperm function, nevertheless the level of evidence is limited. This suggests the effectiveness of Maca but more systematic clinical studies are needed.

\section{Acknowledgements}

None.

\section{Conflict of interest}

The author declares no conflict of interest.

\section{References}

1. Yábar E, Pedreschi R, Chirinos R, et al. Glucosinolate content and myrosinase activity evolution in three maca (Lepidium meyenii Walp.) ecotypes during preharvest, harvest and postharvest drying. Food Chemistry. 2011;127(4):1576-1583.

2. M S Lee, Lee HW, You S, et al.The use of maca (Lepidium meyenii) to improve semen quality: A systematic review. Maturita. 2016;92:64-69.

3. Y Ohta, Kawate N, Inaba T, et al. Feeding hydroalcoholic extract powder of Lepidium meyenii (maca) enhances testicular gene expression of $3 \beta$-hydroxysteroid dehydrogenase in rats. Andrologia. 2017.10.1111/ and.12792.

4. Gustavo F. The transillumination technique as a method for the assessment of spermatogenesis using medicinal plants:The effect of extracts of black maca (Lepidium meyenii) and camucamu (Myrciariadubia) on stages of the spermatogenic cycle in male rats. Toxicology Mechanisms and Methods. 2013;23(8).

5. G F Gonzalez, Cordova A, Gonzales C, et al. Lepidium meyenii (Maca) improved semen parameters in adult men. Asian J Androl. 2001;3(4):301-303.

6. S Yucra, M Gasco, J Rubio, et al. Effect of different fractions from hydroalcoholic extract of Black Maca (Lepidium meyenii) on testicular function in adult male rats. Fertil Steril. 2008;89(5 Suppl):1461-1467.

7. I Melnikovova, Tomas Fait, Michaela Kolarova, et al. Effect of Lepidium meyenii Walp. on Semen Parameters and Serum Hormone Levels in Healthy Adult Men:A Double-Blind, Randomized, Placebo-Controlled Pilot Study. Hindawi. Evidence-Based Complementary and Alternative Medicine. 2015;2015:324369.

8. Yoshida K, Ohta Y, Kawate N, et al. Long-term feeding of hydroalcoholic extract powder of Lepidium meyenii (maca) enhances the steroidogenic ability of Leydig cells to alleviate its decline with ageing in male rats. Andrologia. 2017.

9. C Gonzalez, J Rubio, Gasco M, et al. Effect of short-term and long-term treatments with three ecotypes of Lepidium meyenii (MACA) on spermatogenesis in rats. J Ethnopharmacol. 2006;103(3):448-454.

10. A Rodríguez Huamán. Antioxidant and Neuroprotector effect of Lepidium meyenii (MACA) methanol leaf extract against 6-hydroxy dopamine (6-OHDA)-induced toxicity in PC12 cells. Toxicol Mech Methods. 2017;27(4):279-285.

11. H O Meissner, P Mrozikiewicz, T Bobkiewicz-Kozlowska, et al. Hormone-Balancing Effect of Pre-Gelatinized Organic Maca (Lepidium peruvianum Chacon):(I) Biochemical and Pharmacodynamic Study on Maca using Clinical Laboratory Model on Ovariectomized Rats. Int $J$ Biomed Sci. 2006;2(3):260-272.

12. H O Meissner, H Reich-Bilinska, A Mscisz, et al. Therapeutic effects of pre-gelatinized maca (lepidium peruvianum chacon) used as a non-hormonal alternative to hrt in perimenopausal women - clinical pilot study. Int J Biomed Sci. 2006;2(2):143-159.

13. Uchiyama F, Jikyo T, Takeda R, et al. Lepidium meyenii (Maca) enhances the serum levels of luteinising hormone in female rats. $J$ Ethnopharmacol. 2013;151(2):897-902.

14. The International Association for Contract and Commercial Management. Evaluation of a new class 1 substance:Lepidium meyenii walpers. 2006 .

15. Rosales-Hartshorn M. Maca: Botanical medicine from the andes. $A d v$ Food Technol Nutr Sci Open J. 2015;1(2):1-6. 